\title{
Hard X-ray timing and spectral properties of PSR B0540-69
}

\author{
J. de Plaa ${ }^{1,2}$, L. Kuiper ${ }^{1}$, and W. Hermsen ${ }^{1}$ \\ 1 SRON National Institute for Space Research, Sorbonnelaan 2, 3584 CA Utrecht, The Netherlands \\ e-mail: L.M.Kuiper@sron.nl; W.Hermsen@sron.nl \\ 2 Astronomical Institute, Utrecht University, PO Box 80000, 3508 TA Utrecht, The Netherlands
}

Received 19 November 2002 / Accepted 9 January 2003

\begin{abstract}
We report the hard X-ray properties of the young Crab-like LMC pulsar PSR B0540-69, using archival RXTE PCA (2-60 keV) and RXTE HEXTE (15-250 keV) data. Making use of the very long effective exposure of $684 \mathrm{ks}$, we derived a very detailed master pulse profile for energies $2-20 \mathrm{keV}$. We confirm the broad single-pulse shape with a dip in the middle and with a significant fine structure to the left of the dip. For the first time pulse profiles in the $10-50 \mathrm{keV}$ energy interval are shown. Remarkably, the coarse pulse shape is stable from the optical up to X-ray energies analogous to the case of the Crab pulsar (PSR B0531+21). The profiles can be described with two Gaussians with a phase separation of $\sim 0.2$; the distribution of the ratios between the two components from the optical to the X-ray range is consistent with being flat. Therefore we cannot conclude that the profile consists of two distinct components. We also derived a new total pulsed spectrum in the $\sim 0.01-50 \mathrm{keV}$ range in a consistent analysis including also archival ROSAT PSPC (0.01-2.5 keV) data. This spectrum cannot be described by a single power-law, but requires an additional energy dependent term. The bending of the spectrum around $10 \mathrm{keV}$ resembles that of the Crab pulsar spectrum. Although model calculations using Outer Gap scenarios could probably explain the highenergy characteristics of PSR B0540-69 as they successfully do for the Crab, our measurements do not entirely agree with the latest calculations by Zhang \& Cheng (2000). The small discrepancies are likely to be caused by uncertainties in the pulsar's geometry.
\end{abstract}

Key words. pulsars: individual: PSR B0540-69 - pulsars: individual: PSR B0531+21 - pulsars: individual: PSR B1509-58 stars: neutron - X-rays: stars

\section{Introduction}

PSR B0540-69 is a young Crab-like pulsar located in the Large Magellanic Cloud (LMC) and was discovered at X-rays by Seward et al. (1984) who used the Einstein X-ray Observatory. Like the Crab it is embedded in a bright synchrotron wind nebula which was confirmed in the optical waveband (Chanan et al. 1984) shortly after the discovery of the pulsar. The rotational characteristics of PSR B0540-69 resemble those of the Crab pulsar as well, therefore their spin-down ages are of the same order of magnitude, $\sim 10^{3} \mathrm{yr}$.

The pulse profile of PSR B0540-69, however, is considerably different from that of the Crab. The Crab profile shows two sharp peaks from optical to high-energy gamma-rays (for the most recent high-energy picture from soft X-rays up to highenergy gamma-rays see Kuiper et al. 2001), while the pulse profile of PSR B0540-69 consists mainly of a broad asymmetric pulse with indications for fine structure (Middleditch \& Pennypacker 1985; Boyd et al. 1995; Seward et al. 1984). Most recently, Mineo et al. (1999) described the pulse shape as nearly sinusoidal with a minor structure on the left side of the maximum on the basis of higher-statistic BeppoSAX data.

Send offprint requests to: J. de Plaa, e-mail: j.de.plaa@sron.nl
Hirayama et al. (2002) analysed all available ASCA data on this source. They confirmed the broad profile and discussed a hump on the leading wing of the profile as a possible interpulse, analogous to the case of the Crab.

Measurements of the total pulsed spectrum of PSR B0540-69 between 0.2 and $10 \mathrm{keV}$ by the Chandra X-ray Observatory suggest a hard spectrum with a photon spectral index of $1.83 \pm 0.13$ (Kaaret et al. 2001), consistent with the BeppoSAX (Mineo et al. 1999) and ASCA (Hirayama et al. 2002) spectral findings. At soft X-rays (ROSAT 0.1$2.4 \mathrm{keV}$ ) Finley et al. (1993) reported an index $1.3 \pm 0.5$ (90\% errors), statistically consistent with the indices measured above $2 \mathrm{keV}$. If the pulsed spectrum would extend from medium energy X-rays with the same power-law index into the hard X-ray or soft gamma-ray regime, then pulsed emission should have been detected by the BATSE (20-600 keV) and OSSE (50 keV-10 MeV) instruments aboard the Compton Gamma Ray Observatory (CGRO). However, no detection of pulsed soft gamma-ray emission from PSR B0540-69 has been reported sofar (Hertz et al. 1995; Wilson et al. 1993).

The Rossi X-ray Timing Explorer (RXTE) archive contains a huge amount of hard X-ray data with PSR B0540-69 in the field of view. A part of these data had been used by 
Table 1. List of all RXTE observations used in this study with PSR B0540-69 within 30' of the pointing direction. The exposures mentioned are effective values screened for Earth occultations, SAA passages and contaminating particles.

\begin{tabular}{llrr}
\hline \hline Obs. ID & Target & $\begin{array}{r}\text { Offset } \\
\left({ }^{\prime}\right)\end{array}$ & $\begin{array}{r}\text { Exposure } \\
(\mathrm{ks})\end{array}$ \\
\hline 10206 & PSR B0540-69 & 0.04 & 39.1 \\
10218 & SN1987A & 25.30 & 74.1 \\
10250 & LMC X-1 & 24.80 & 33.4 \\
20188 & LMC X-1 & 24.80 & 234.8 \\
30087 & LMC X-1 & 24.77 & 73.5 \\
40139 & PSR J0537-69 & 15.96 & 109.0 \\
50103 & PSR J0537-69 & 15.94 & 120.0 \\
\hline
\end{tabular}

Zhang et al. (2001) to provide an accurate measurement of the spin frequency of the pulsar and its first and second derivatives by phase-linking on a limited time interval. In this paper we present the hard X-ray characteristics of PSR B0540-69 using all these available RXTE Proportional Counter Array (PCA, 2-60 keV) and RXTE High Energy X-ray Timing Experiment (HEXTE, 15-250 keV) data. In order to augment the spectral coverage towards lower energies we also used soft X-ray data $(\sim 0.01-2.5 \mathrm{keV})$ from the ROSAT PSPC in a consistent analysis.

\section{Observations and data}

\subsection{RXTE PCA}

The PCA (Jahoda 1994) consists of five Proportional Counter Units (PCU) sensitive in the $\sim 2-60 \mathrm{keV}$ range with an energy resolution less than $18 \%$ at $6 \mathrm{keV}$ and a large field of view of $1^{\circ}$ FWHM. Each PCU has three xenon layers which provide the basic scientific data. When running in Good Xenon configuration each photon captured in a xenon layer is registered with a timing resolution of $1 \mu \mathrm{s}$. The individual triggers do not contain any spatial information.

The PCA observed the LMC many times with PSR B0540-69 in its large field of view. We analysed archival data from observations performed in the Good Xenon configuration with offset angles less than $30^{\prime}$ and durations longer than $\sim 5 \mathrm{ks}$ (see Table 1), resulting in a total dataset containing $684 \mathrm{ks}$ of exposure, after screening for Earth occultations, South Atlantic Anomaly (SAA) passages and enhanced background due to high-energy particles. We used this exceptionally long exposure of PSR B0540-69 for our timing analysis. To boost the signal-to-noise ratio for this weak source we selected the photons registered in the first xenon layer of the PCUs.

\subsection{RXTE HEXTE}

The HEXTE instrument (Rothschild et al. 1998) aboard RXTE consists of two independent detector clusters (A and B) each containing $4 \mathrm{Na}(\mathrm{Tl}) / \mathrm{CsI}(\mathrm{Na})$ phoswich scintillation detectors passively collimated to a $1^{\circ} \mathrm{FWHM}$ field of view and co-aligned with the PCA. The instrument is sensitive to photons with energies in the energy range $15-250 \mathrm{keV}$ with an energy resolution of about $15.4 \%$ at $60 \mathrm{keV}$. The net open area of the 8 detectors is $1600 \mathrm{~cm}^{2}$. The events can be tagged with a maximum time resolution of $7.6 \mu \mathrm{s}$. In its default operation mode the field of view of each cluster is switched on and off source to provide instantaneous background measurements reducing the effective source-on exposure by roughly a half. Due to high-energy particle events the overall performance of the instrument is degraded resulting in a dead-time fraction of about $40 \%$. When the source of interest is observed off-axis the response is further reduced by the collimator/detector assembly. For a source observed $30^{\prime}$ off-axis the sensitive area is reduced to about 0.5 of its on-axis value.

In the spectral analysis of HEXTE data all these effects which reduce the effective sensitive area are taken into account. Due to the co-alignment of HEXTE with the PCA the same observations have been used as for the PCA. For the spectral analysis HEXTE data from all observations listed in Table 1 have been used, not limited to those observations falling within the stable gain period used in the PCA spectral analysis.

The total dead-time and off-axis corrected Cluster-A and B on-source (=PSR B0540-69) exposures are $248.4 \mathrm{ks}$ and $260.7 \mathrm{ks}$, respectively.

\subsection{ROSAT PSPC}

To extend the spectral coverage to the soft X-ray regime we also included ROSAT PSPC data in our study. The PSPC is a gas-filled imaging proportional counter which operates in photon counting mode (Pfeffermann \& Briel 1986). It is sensitive over the $0.01-2.5 \mathrm{keV}$ energy band. The energy resolution is $\Delta E=0.45 E^{0.5}$. The events are tagged on board with a precision of $130 \mu \mathrm{s}$, but the erratic spacecraft clock makes absolute timing difficult.

We could identify in the ROSAT archive 3 PSPC observation sequences with PSR B0540-69 nearly on-axis. The observation identifiers are RP400052N00 (PSPC-B), RP150044N00 (PSPC-C) and RP400133N00 (PSPC-B), with effective exposure times of $8.5 \mathrm{ks}, 5.1 \mathrm{ks}$ and $1.7 \mathrm{ks}$, respectively.

\section{Timing analysis}

For all selected photons measured with the RXTE PCA with energies above $2 \mathrm{keV}$ barycentric corrections to their arrival times have been derived using the FTOOLS program fasebin (Blackburn 1995) and a source position of $\alpha_{2000}=$ $05^{\mathrm{h}} 40^{\mathrm{m}} 11^{\mathrm{s}} .221$ and $\delta_{2000}=-69^{\circ} 19^{\prime} 54^{\prime \prime} .98$ (Kaaret et al. 2001). The ephemeris used to derive the pulse profile is presented in Table 2. This ephemeris predicts accurately the instantaneous rotation frequency of the source for the epochs of our observations. However, it is not a phase connected timing solution.

For each observation contributing to our total data set of $684 \mathrm{ks}$ we derived a pulse profile. Because the ephemeris is not a phase connected solution for long timescales, we cannot combine the individual profiles directly. Therefore, we correlated all pulse profiles first with a profile template based on the longest data block and later again with the result of the 
Table 2. Ephemeris of PSR B0540-69 determined by Kaaret et al. (2001).

\begin{tabular}{ll}
\hline \hline Parameter & Value \\
\hline Val. range (MJD) & $46992-51421$ \\
$t_{0}(\mathrm{MJD})$ & 47700.0 \\
$v_{0}(\mathrm{~Hz})$ & $19.8593584982(40)$ \\
$\dot{v}_{0}\left(10^{-10} \mathrm{~Hz} \mathrm{~s}^{-1}\right)$ & $-1.8894081(7)$ \\
$\ddot{v}_{0}\left(10^{-21} \mathrm{~Hz} \mathrm{~s}^{-2}\right)$ & $3.7425(43)$ \\
$\alpha_{2000}$ & $05^{\mathrm{h}} 40^{\mathrm{m}} 11^{\mathrm{s}} .221$ \\
$\delta_{2000}$ & $-69^{\circ} 19^{\prime} 54^{\prime \prime} .98$
\end{tabular}

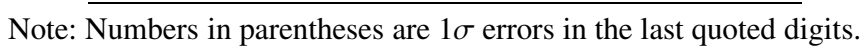

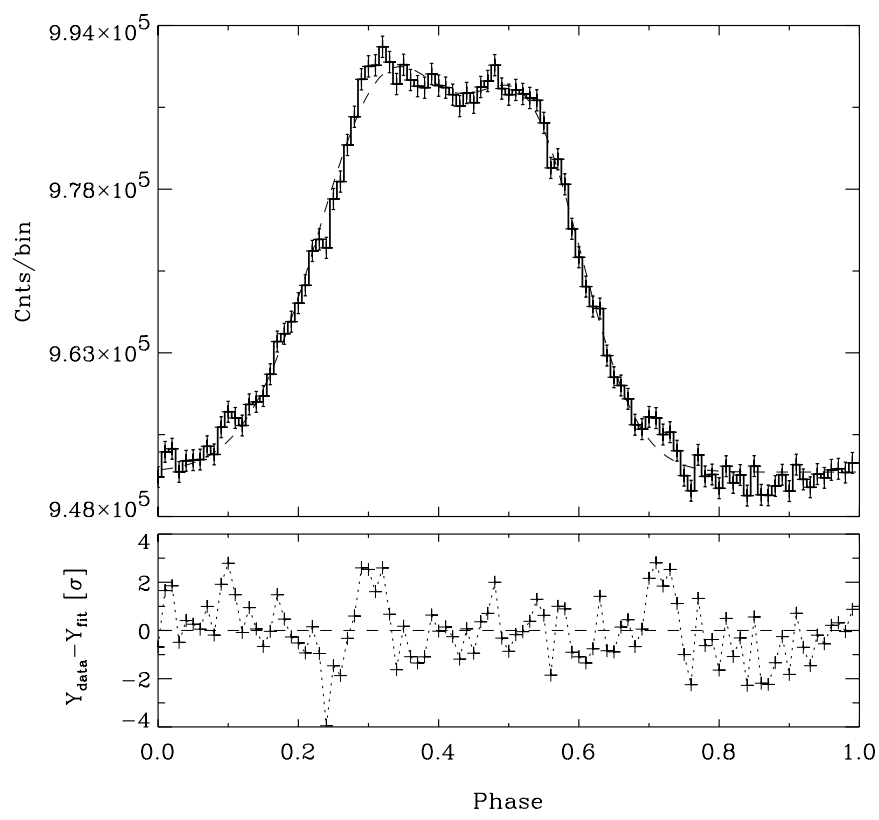

Fig. 1. Master pulse profile of PSR B0540-69 derived from the total $684 \mathrm{ks}$ RXTE PCA data set for energies $2-20 \mathrm{keV}$. The broken line shows the best fit empirical model consisting of two symmetric Gaussian profiles separated $\sim 0.2$ in phase. The lower figure shows the distribution of the residuals in number of $\sigma$ 's.

aligned combination to obtain the final profile. The statistical uncertainties in the estimates of the phase shifts varied approximately over the range $0.6-3 \%$.

Figure 1 shows the resulting pulse profile $(2-20 \mathrm{keV})$ of PSR B0540-69 for the total screened exposure of $684 \mathrm{ks}$. The statistical accuracy of this profile is at least an order of magnitude higher than for earlier published profiles (e.g. Eikenberry et al. 1998; Mineo et al. 1999; Hirayama et al. 2002) and the genuine profile becomes more clearly visible: A single broad pulse with a clear dip at the top. Such a shape could be the result of two narrower pulses separated $\sim 0.2$ in phase. To investigate this further, we attempted to describe the shape of the profile as the sum of two symmetrical Gaussians on top of a flat background, by making a fit to the measured profile with as free parameters the widths, positions and amplitudes of the gaussians. The resulting best fit is shown in Fig. 1 (positions: $p_{1}=0.330 \pm 0.004, p_{2}=0.531 \pm 0.003$; widths: $\sigma_{1}=0.102 \pm 0.003, \sigma_{2}=0.079 \pm 0.002$ ) with underneath the fit residuals expressed in number of $\sigma$ 's. The overall fit looks good, particularly for the component with maximum at phase 0.531 , but there are a few phase intervals in which there appear significant deviations from this empirical model $\left(\chi_{\mathrm{r}}^{2}\right.$ of fit: $167.3 / 93 ; P\left(\chi^{2}>167.3 \mid 93\right)=3.65 \times 10^{-6}(4.7 \sigma$ dev. $\left.)\right)$. The most significant positive excess is around phase 0.3 (near the position of one of the Gaussians) reaching a significance of $5.4 \sigma$, proving that the top of the broad profile has significant fine structure. The positive excesses far in the wings at phases around 0.1 and 0.7 reach significances of 3.6 and $4.0 \sigma$, respectively. We do not consider these latter deviations significant, because there was no a priori reason to select these phases (no single trial). Hirayama et al. (2002) discussed a hump in the ASCA profile at a phase consistent with our excess around phase 0.1 , as a possible separate interpulse in analogy to the Crab profile. With our better statistics we do not confirm the detection of this hump. Finally, the negative excursion at phase 0.24 , together with the positive excess at phase 0.3 suggest that the leading wing of the profile is steeper than fitted with the Gaussian profile.

The high counting statistics in the PCA data allow us to construct significant pulse profiles in differential energy windows from $2 \mathrm{keV}$ up to $30 \mathrm{keV}$ (Figs. $2 \mathrm{~d}$ to j), showing the first profiles above $10 \mathrm{keV}$. Up to $12 \mathrm{keV}$ the statistical accuracy is very high and some fine structure on top of the broad profile seems to be consistently present in successive energy windows, most notably the sharp maximum at phase $\sim 0.3$.

Figure 2 also shows pulse profiles we derived from RXTE HEXTE data at higher energies and from ROSAT data at lower energies. We could construct the HEXTE profiles of PSR B0540-69 by following the same analysis steps for the same observation windows as was performed for the PCA timing analysis, applying the phase shifts determined in the correlation analysis of the high-statistics PCA profiles to the low-statistics HEXTE profiles. This allowed us to reveal the PSR B0540-69 pulse profile up to $48 \mathrm{keV}$. The ROSAT profiles are derived using the data reported earlier in Sect. 2.3, and the phase alignment was determined by cross correlating the PCA master profile with the total ROSAT profile. Finally, the optical PSR B0540-69 pulse profile from the High Speed Photometer (HSP) aboard the Hubble Space Telescope (HST) (Boyd et al. 1995) is shown. The optical profile shape is strikingly similar to the PCA X-ray profile. Therefore, we choose to align also the optical profile by cross correlation. We note, however, that Ulmer et al. (1999) report in a preliminary communication that there is a phase shift of 0.17 between the optical and the X-ray pulse, the optical arriving earlier. We would like to see this result confirmed.

At a first glance it is not obvious, due to the varying statistics, whether the pulse shape varies from the optical to the hard X-ray windows. Therefore, we fitted now all profiles in Fig. 2 with the empirical model of two Gaussian profiles with the positions and the widths fixed to the best-fit values shown in Fig. 1 and leaving the normalisations free. We verified that all fits are good with reduced $\chi^{2}$ values ranging between 0.93 and 1.32 (98 d.o.f.), except for the optical profile (18 d.o.f.). 


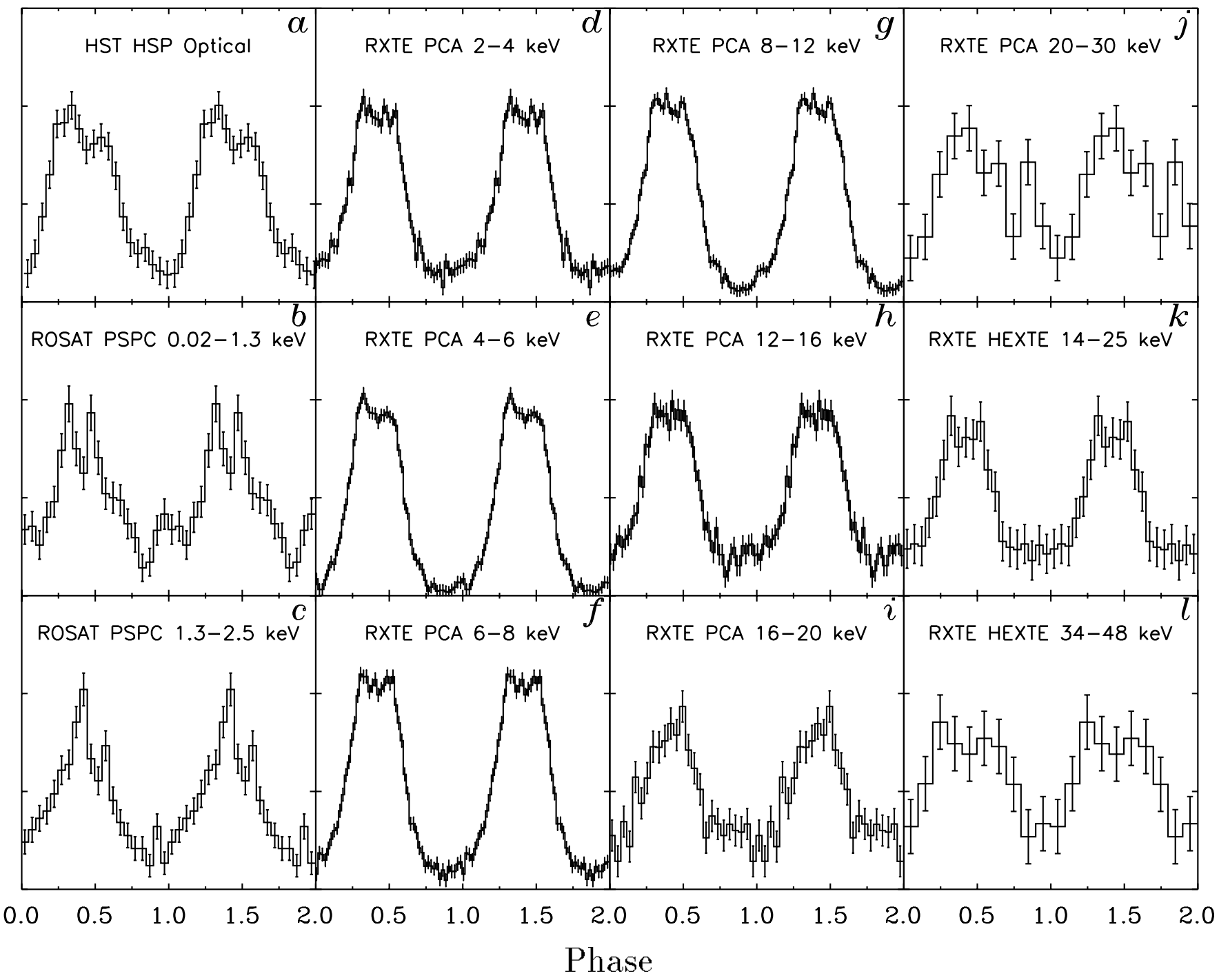

Fig. 2. Pulse profiles of PSR B0540-69 from the optical window to hard X-rays. a) Optical profile as observed by Boyd et al. (1995) with Hubble's High Speed Photometer (HSP). b)-c) ROSAT PSPC, energy ranges 0.02-1.3 and 1.3-2.5 keV. d)-j) RXTE PCA, energy ranges 2-4, 4-6, 6-8, 8-12, 12-16, 16-20 and 20-30 keV. k)-l) RXTE HEXTE, energy ranges 14-25 and 34-48 keV. The RXTE HEXTE lightcurve for energies $25-34 \mathrm{keV}$ is not shown since it does not give a significant signal due to high instrumental background. The ROSAT and HST profiles have been aligned in phase to the RXTE profiles by cross correlation.

Only this optical profile appears to be somewhat broader than the X-ray profiles. Figure 3 shows the ratio of the counts in the two Gaussian components from the optical regime to the hard $\mathrm{X}$-rays. This ratio appears to be surprisingly constant, underlining the stability of the profile shape and showing that the spectra of the two empirical components are within the statistics the same. Therefore, we cannot conclude that the broad and structured pulse profile of PSR B0540-69 is the sum of two distinct components with different spectra.

\section{Spectral analysis}

Unfortunately, the total RXTE PCA data set of $684 \mathrm{ks}$ is not uniform in energy calibration, because the gain settings on the PCA instrument have been changed a number of times since the launch of RXTE. For the derivation of the total pulsed spectrum of PSR B0540-69 we selected therefore the observations performed in the third and longest gain epoch (April 15, 1996, 23:05-March 22, 1999, 17:37), to obtain a dataset with a constant channel-to-energy relation with total exposure of $462 \mathrm{ks}$. For each observation in this data set we derived the pulse profile and the response corrected for the offset angle. If an observation contained one or more time intervals in which the number or the order of active PCUs was different, then these intervals were treated as separate data blocks. All offset-corrected response files were summed using weighing factors $\left(f_{i}\right)$ as calculated in Eq. (1), where $N_{\mathrm{pcu}, i}$ is the number of PCUs on in data block $i, t_{i}$ is the exposure of data block $i$ and $T$ is the total exposure of the selected (third epoch) dataset.

$f_{i}=\frac{N_{\mathrm{pcu}, i} t_{i}}{5 T}$

Pulse profiles in 14 differential energy ranges between 3.4 and $30.2 \mathrm{keV}$ for the $462 \mathrm{ks}$ data set were derived following the same procedure as explained in the timing analysis. 


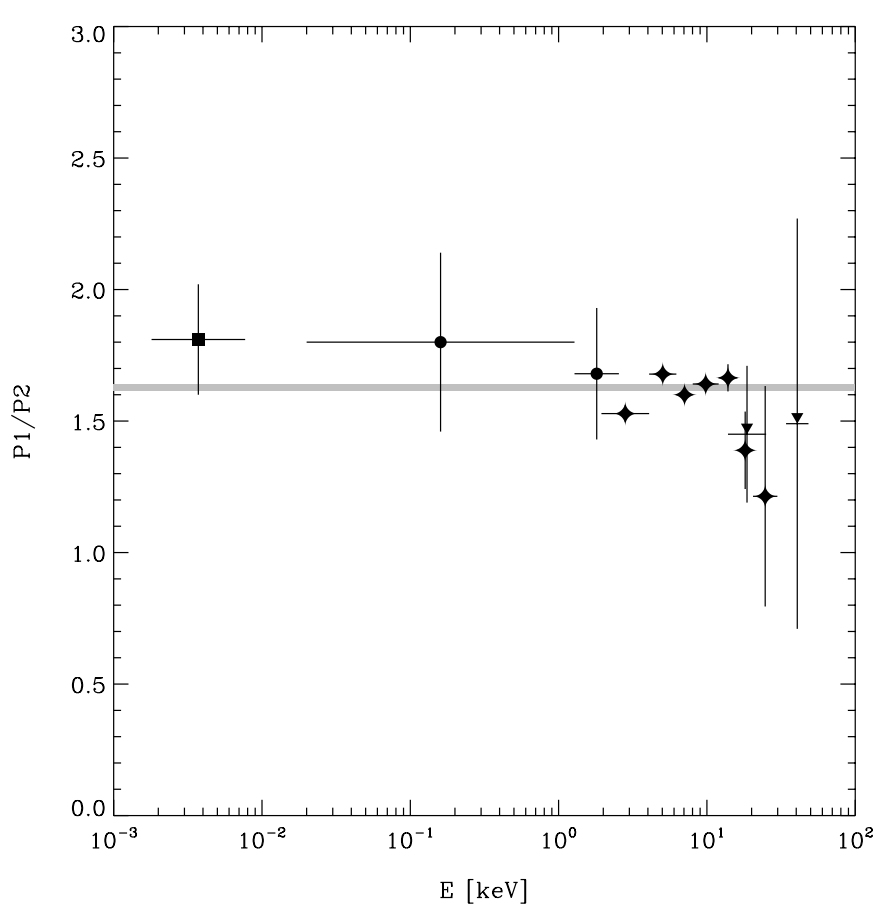

Fig. 3. Ratio of the pulsed (excess) counts assigned to the two Gaussians components of the model fit to the profiles shown in Fig. 2. This distribution is consistent with being flat with a $\mathrm{P} 1 / \mathrm{P} 2$ value of $1.63 \pm 0.01$ with a $\chi_{\mathrm{r}}^{2}$ of $21.66 / 11$ resulting in a random probability of $2.7 \%$. Data points are derived from the optical HST HSP (square), ROSAT PSPC (circle), RXTE PCA (diamond) and RXTE HEXTE (triangle) observations.

In order to determine the excess counts in the broad pulse profiles for all 14 differential energy ranges, we fitted these profiles with the shape of the best-model fit shown in Fig. 1. Again, we verified that the model fits were statistically in agreement with the data for all energy intervals. Using the numbers of excess counts and the derived response values we can derive the PSR B0540-69 pulsed spectrum measured by the PCA.

For the ROSAT and RXTE HEXTE data we performed a consistent spectral analysis. Also for the profiles measured by these instruments we determined the pulsed excess counts by making fits with the empirical model. The resulting total pulsed X-ray spectrum of PSR B0540-69 is shown in Fig. 4; the flux values are given in Table 3. From the plot it is evident that the X-ray spectrum has not the shape of a single power-law. A single absorbed power-law fit to just the ROSAT PSPC and RXTE PCA data renders a photon index of $1.845 \pm 0.004$, fixing the hydrogen column density $\left(N_{\mathrm{H}}\right)$ at $4.6 \times 10^{21} \mathrm{~cm}^{-2}$ (Kaaret et al. 2001). This value is consistent with earlier measurements: $1.83 \pm 0.13$ (Kaaret et al. 2001), $1.94 \pm 0.03$ (Mineo et al. 1999) and $1.3 \pm 0.5$ (Finley et al. 1993). However, our reduced $\chi^{2}$ is unacceptably high with a value of 4.75 with 20 d.o.f., rejecting this simple shape. Therefore we have fitted the data with an absorbed power-law model with an energy dependent index:

$F\left(E_{\gamma}\right)=\mathrm{e}^{-N_{\mathrm{H}} \cdot \sigma} \alpha E_{\gamma}^{-\left(\beta+\gamma \ln E_{\gamma}\right)}$.

In the fit the parameters $\alpha, \beta$ and $\gamma$ were kept free and $N_{\mathrm{H}}$ was fixed again at $4.6 \times 10^{21} \mathrm{~cm}^{-2}$.
Table 3. Flux values derived from the ROSAT PSPC, RXTE PCA and RXTE HEXTE data (see Fig. 4). In this table we give $2 \sigma$ upper limits.

\begin{tabular}{|c|c|}
\hline $\begin{array}{l}\text { Energy interval } \\
\quad(\mathrm{keV})\end{array}$ & $\begin{array}{c}\text { Flux } \\
\left(\text { photons s} \mathrm{s}^{-1} \mathrm{~cm}^{-2} \mathrm{keV}^{-1} \text { ) }\right.\end{array}$ \\
\hline \multicolumn{2}{|c|}{ ROSAT PSPC } \\
\hline $0.01-0.33$ & $<3 \times 10^{-2}$ \\
\hline $0.33-0.63$ & $<5.8 \times 10^{-3}$ \\
\hline $0.64-0.97$ & $(2.6 \pm 0.4) \times 10^{-3}$ \\
\hline $0.97-1.28$ & $(1.8 \pm 0.2) \times 10^{-3}$ \\
\hline $1.28-1.60$ & $(1.2 \pm 0.1) \times 10^{-3}$ \\
\hline $1.60-1.93$ & $(8.0 \pm 1.0) \times 10^{-4}$ \\
\hline $1.93-2.25$ & $(7.0 \pm 1.4) \times 10^{-4}$ \\
\hline $2.25-2.55$ & $(7.1 \pm 2.6) \times 10^{-4}$ \\
\hline \multicolumn{2}{|c|}{ RXTE PCA } \\
\hline $3.37-4.08$ & $(2.31 \pm 0.07) \times 10^{-4}$ \\
\hline $4.08-4.79$ & $(1.83 \pm 0.05) \times 10^{-4}$ \\
\hline $4.79-5.51$ & $(1.49 \pm 0.04) \times 10^{-4}$ \\
\hline $5.51-6.58$ & $(1.03 \pm 0.02) \times 10^{-4}$ \\
\hline $6.58-7.66$ & $(7.63 \pm 0.15) \times 10^{-5}$ \\
\hline $7.66-8.74$ & $(5.65 \pm 0.12) \times 10^{-5}$ \\
\hline $8.74-10.2$ & $(4.28 \pm 0.09) \times 10^{-5}$ \\
\hline $10.2-12.0$ & $(3.11 \pm 0.07) \times 10^{-5}$ \\
\hline $12.0-14.2$ & $(2.32 \pm 0.08) \times 10^{-5}$ \\
\hline $14.2-16.4$ & $(1.76 \pm 0.09) \times 10^{-5}$ \\
\hline $16.4-19.0$ & $(10.0 \pm 0.1) \times 10^{-5}$ \\
\hline $19.0-22.3$ & $(6.6 \pm 1.2) \times 10^{-6}$ \\
\hline $22.3-25.7$ & $(7.3 \pm 1.8) \times 10^{-6}$ \\
\hline $25.7-30.2$ & $(4.3 \pm 2.3) \times 10^{-6}$ \\
\hline \multicolumn{2}{|c|}{ RXTE HEXTE } \\
\hline $13.9-25.1$ & $(8.1 \pm 0.7) \times 10^{-6}$ \\
\hline $25.1-34.2$ & $(1.8 \pm 1.4) \times 10^{-6}$ \\
\hline $34.2-48.4$ & $(2.6 \pm 0.7) \times 10^{-6}$ \\
\hline $48.4-75.4$ & $<1.5 \times 10^{-6}$ \\
\hline $75.4-102$. & $<7.8 \times 10^{-7}$ \\
\hline $102 .-157$. & $<6.8 \times 10^{-7}$ \\
\hline 157.-268. & $<9.6 \times 10^{-7}$ \\
\hline
\end{tabular}

This resulted in a $\beta$ of $1.360 \pm 0.005$ and $\gamma$ of $0.143 \pm 0.003$, which means that the photon index softens at higher energies (for example at $1 \mathrm{keV}: \sim 1.35,2 \mathrm{keV}: \sim 1.56,4 \mathrm{keV}: \sim 1.76$ and $8 \mathrm{keV}: \sim 1.95)$. With a $\chi_{\mathrm{r}}^{2}$ of $31.0 / 19$ the model fits reasonably well to the measured spectrum. For energies between 20 and $50 \mathrm{keV}$, the measured shape is poorly defined, and the flux values of the PCA and HEXTE are also consistent with a power-law with index 2 .

\section{Summary and discussion}

This study of the hard X-ray characteristics of the young spin-down powered pulsar PSR B0540-69 using archival RXTE PCA/HEXTE and ROSAT PSPC observations resulted 


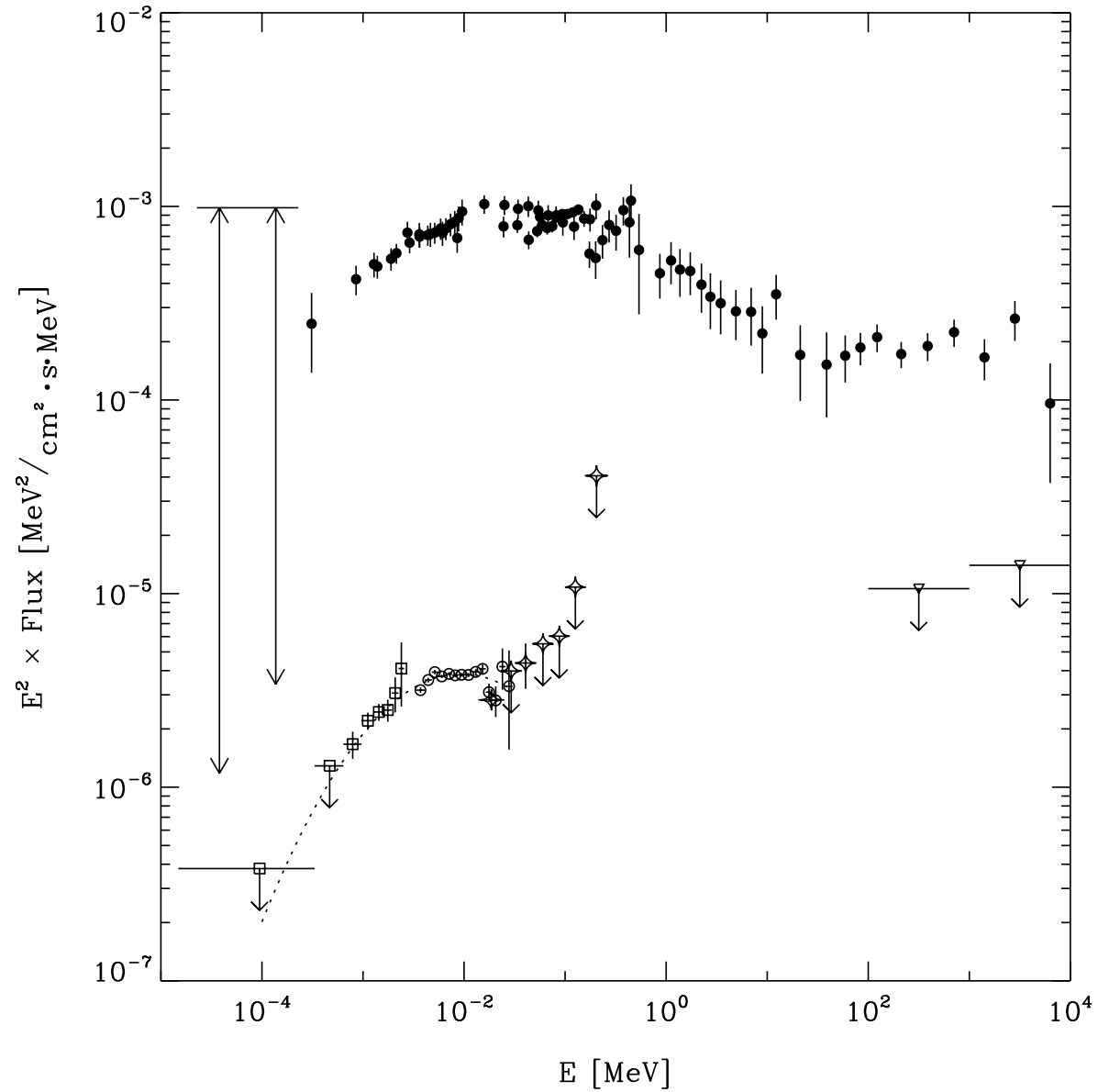

Fig. 4. Total pulsed spectrum of the Crab pulsar and PSR B0540-69 for X-ray energies above $0.01 \mathrm{keV}$. Crab (top): Flux measurements taken from Kuiper et al. (2001). PSR B0540-69 (bottom): Flux measurements and upper limits from ROSAT PSPC (open squares, 0.01-2.5 keV), RXTE PCA (open circles, 3.4-30 keV) and RXTE HEXTE (open diamonds, 14 $300 \mathrm{keV}$ ) are shown, as well as upper limits at high-energy $\gamma$-rays from CGRO EGRET (Thompson et al. 1994). The sizes of the arrows on the left indicate the shift in flux $( \pm 1 \sigma)$ to put the Crab pulsar at the LMC distance. We used $2.0 \pm 0.5 \mathrm{kpc}$ for the $\mathrm{Crab}$ and $49.4 \pm 3.4 \mathrm{kpc}$ for the PSR B0540-69 (LMC) distance. in a detection of this pulsar up to $\sim 50 \mathrm{keV}$. For the first time pulse profiles above $10 \mathrm{keV}$ are shown. Furthermore, we derived the total pulsed spectrum of PSR B0540-69 in a consistent analysis from $\sim 0.01-50 \mathrm{keV}$.

Because of the high statistics we were able to obtain a very detailed master pulse profile in the $2-20 \mathrm{keV}$ range, which could reasonably be described by the sum of two symmetrical Gaussians. Remarkably, this double Gaussian shape could be well fitted to all shown X-ray pulse profiles and resembles very much the optical profile which is, however, somewhat broader. The distribution of the ratios between the two Gaussian components as a function of energy appears to be consistent with being flat. Therefore we could not conclude that the pulse consists of two distinct Gaussian components with different spectra.

The derived total pulsed spectrum of PSR B0540-69 clearly shows a bend towards higher energies and cannot be described by a single power-law. We proposed and fitted an energy dependent power-law model to the data, successfully describing the curved spectral shape up to $20 \mathrm{keV}$. Between 20 and $50 \mathrm{keV}$ the measured flux shape is also consistent with a power-law with index 2.

Directly after the discovery of PSR B0540-69 its timing properties, apart from the pulse profile, were found to be very Crab-like (Seward et al. 1984). If we compare our results to the characteristics of the Crab pulsar we see some remarkable additional similarities. Even though the Crab and PSR B0540-69 show different pulse profiles (Crab: two sharp peaks, PSR B0540-69: one broad structured pulse), for both the coarse shapes of the profiles remain stable from optical to hard X-rays. The second Crab-like pulsar (PSR B1509-58) also shows a very stable single broad pulse from soft X-rays up to MeV $\gamma$-rays (Kuiper et al. 1999; Cusumano et al. 2001), but pulsed optical emission has not been found sofar.

Furthermore, the shape of the total pulsed spectrum of PSR B0540-69 looks strikingly similar to that of the Crab in the $\sim 0.01-50 \mathrm{keV}$ range. In Fig. 4 the two total pulsed spectra are plotted in $E^{2} F(E)$ representation. The bending of the spectrum around $10 \mathrm{keV}$ appears to occur similarly in both spectra. The intrinsic X-ray luminosities are comparable as well, within the uncertainties caused by the errors in the Crab distance and the absolute calibrations of the RXTE PCA and HEXTE detectors.

Next to PSR B0540-69 and the Crab pulsar, PSR B1509-58 shows a similar spectral behaviour at X-ray $/ \gamma$-ray energies (Kuiper et al. 1999; Cusumano et al. 2001), although its spectrum appears to reach its maximum luminosity at higher energies (10-30 MeV) relative to the Crab and PSR B0540-69. These three young $\left(\lesssim 1.6 \times 10^{3} \mathrm{yr}\right) \mathrm{X}$-ray $/ \gamma$-ray pulsars exhibit different spectral behaviours compared to older $\gamma$-ray pulsars like, for example, the Vela pulsar and Geminga. Older $\gamma$-ray pulsars show a hard high-energy spectrum with a turn-over at $\mathrm{GeV}$ energies. Therefore, the observed spectral shape for the three young pulsars might be characteristic for very young pulsars in general. 
The models that have been proposed to explain high-energy emission from highly magnetised pulsars, can be classified into two distinct classes, namely Polar Cap (e.g. Sturrock 1971; Daugherty \& Harding 1994) and Outer Gap models (e.g. Cheng et al. 1986a; Cheng et al. 1986b; Chiang \& Romani 1994; Romani \& Yadigaroglu 1995; Cheng et al. 2000). Outer gap models seem to be most successful in explaining the Crab pulse profile and spectrum as well as the optical polarisation angle variation with phase (Romani \& Yadigaroglu 1995; Cheng et al. 2000).

As PSR B0540-69 shows predominantly Crab-like characteristics, one may expect that Outer Gap models can explain its profile and spectrum as well. Unfortunately, the geometry of the magnetic inclination $(\alpha)$ and viewing angles $(\zeta)$ cannot be constrained by radio measurements. In a recent model calculation for PSR B0540-69 based on the three dimensional outer magnetosphere model of Cheng et al. (2000), Zhang \& Cheng (2000) chose likely values for these angles $\left(\alpha=50^{\circ}\right.$; $\zeta=76^{\circ}$ ) by constraining the model to the observed radio emission and broad pulse profiles. Their calculations predict a single broad pulse shape in the optical, X-ray and $\gamma$-ray windows and a power-law shaped total pulsed spectrum from the optical up to $\sim 100 \mathrm{keV}$. Above $1 \mathrm{MeV}$ a bend in the spectrum is expected.

Our new hard X-ray findings do not agree entirely with these model calculations. The first difference is in the observed high-resolution pulse profile. We confirmed the broad nature of the pulse, but it also shows a significant dip on the top of the profile with a significant excess to the left of it. Secondly, the observed total pulsed spectrum flattens above $10 \mathrm{keV}$, while the model expects the bend to occur above $100 \mathrm{keV}$. These discrepancies are likely a result of the uncertainties in the pulsar geometry. We note that Zhang \& Cheng (2000) also describe with their Outer Gap model the high energy properties of PSR B1509-58. For slightly different angles $\left(\alpha=60^{\circ}\right.$; $\zeta=75^{\circ}$ ) they predict a broad pulse profile with fine structure over the top, very similar to what we found for PSR B0540-69. This suggests that model calculations for PSR B0540-69 with a slightly larger magnetic inclination angle could reproduce in detail our measured profile. Therefore, optical polarisation measurements are required to constrain the magnetic inclination and viewing angles to model the high-energy emission processes of this young pulsar more accurately.

Earlier, Cheng \& Wei (1995) calculated the high-energy spectrum based on the Outer Gap model of Cheng et al. (1986a,b) assuming that the X-ray and $\gamma$-ray emission is synchrotron self-Compton radiation from secondary $\mathrm{e}^{ \pm}$pairs. They predict a spectral break between about 10 and $100 \mathrm{keV}$ depending on the size of the Outer Gap. A relatively large Outer Gap (gradually increasing with age) could explain the apparent break in the spectrum around $10 \mathrm{keV}$.

A more accurate measurement at higher energies of the total pulsed spectrum of PSR B0540-69 is very important to discriminate the different model calculations. For the INTEGRAL mission $(20 \mathrm{keV}-8 \mathrm{MeV})$ a very long exposure $(2 \mathrm{Ms})$ of the LMC is scheduled. Unfortunately, for the spectral shape we derived in this work INTEGRAL will be able to detect the pulsed signal only up to $\sim 70 \mathrm{keV}$. More importantly, GLAST $(20 \mathrm{MeV}-300 \mathrm{GeV}$ ) should be able to detect pulsed hard $\gamma$-ray emission if the spectrum behaves Crab-like.

Acknowledgements. We wish to thank the High Energy Astrophysics Science Archive Research Center (HEASARC) at NASA/Goddard Space Flight Center for maintaining its online archive service which provided the data used in this research. We also thank Frank Verbunt (Utrecht University) for carefully reading our manuscript.

\section{References}

Blackburn, J. K. 1995, ASP Conf. Ser, 77, 367, http://heasarc.gsfc.nasa.gov/ftools/

Boyd, P. T., van Citters, G. W., Dolan, J. F., et al. 1995, ApJ, 448, 365

Bradt, H. V., Rothschild, R. E., \& Swank, J. H. 1993, A\&AS, 97, 355

Chanan, G. A., Helfand, D. J., \& Reynolds, S. P. 1984, ApJ, 287, L23

Cheng, K., Ho, C., \& Ruderman, M. 1986a, ApJ, 300, 500

Cheng, K., Ho, C., \& Ruderman, M. 1986b, ApJ, 300, 522

Cheng, K., Ruderman, M., \& Zhang, L. 2000, ApJ, 537, 964

Cheng, K. S., \& Wei, D. M. 1995, ApJ, 448, 281

Chiang, J., \& Romani, R. W. 1994, ApJ, 436, 754

Cusumano, G., Mineo, T., Massaro, E., et al. 2001, A\&A, 375, 397

Daugherty, J. K., \& Harding, A. K. 1994, ApJ, 429, 325

Eikenberry, S. S., Fazio, G. G., \& Ransom, S. M. 1998, ApJ, 492, 754

Finley, J. P., Ögelman, H., Hasinger, G., \& Trümper, J. 1993, ApJ, 410,323

Hertz, P., Grove, J. E., Grabelsky, D. A., \& Matz, S. M. 1995, ApJ, 445,351

Hirayama, M., Nagase, F., Endo, T., Kawai, N., \& Itoh, M. 2002, MNRAS, 333, 603

Jahoda, K. 1994, Am. Astrono. Soc. Meet., 26, 894

Kaaret, P., Marshall, H., Aldcroft, T., et al. 2001, ApJ, 546, 1159

Kuiper, L., Hermsen, W., Cusumano, G., et al. 2001, A\&A, 378, 918

Kuiper, L., Hermsen, W., Krijger, J. M., et al. 1999, A\&A, 351, 119

Middleditch, J., \& Pennypacker, C. 1985, Nature, 313, 659

Mineo, T., Cusumano, G., Massaro, E., et al. 1999, A\&A, 348, 519

Pfeffermann, E., \& Briel, U. G. 1986, in X-ray instrumentation in astronomy, Proc. of the Meeting, Cannes, France, December 2-4, 1985 (A87-19722 07-35), Bellingham, WA, Society of Photo-Optical Instrumentation Engineers, 597, 208

Romani, R. W., \& Yadigaroglu, I.-A. 1995, ApJ, 438, 314

Rothschild, R. E., Blanco, P. R., Gruber, D. E., et al. 1998, ApJ, 496, 538

Seward, F., Harnden, F., \& Helfand, D. J. 1984, ApJ, 287, L19

Sturrock, P. 1971, ApJ, 164, 529

Thompson, D. J., Arzoumanian, Z., Bertsch, D. L., et al. 1994, ApJ, 436, 229

Ulmer, M., Park, S., Finley, J., Middleditch, J., \& Codes, J. 1999, Astro. Lett., \& Comm., 38, 29

Wilson, R. B., Fishman, G. J., Finger, M., et al. 1993, AIP Conf. Proc., 280,291

Zhang, L., \& Cheng, K. 2000, A\&A, 363, 575

Zhang, W., Marshall, F., Gotthelf, E., Middleditch, J., \& Wang, Q. 2001, ApJ, 554, L177 